\title{
ALGUNAS CONSIDERACIONES SOBRE LA ALTERNANCIA SUBJUNTIVO-INFINITIVO EN LAS CONSTRUCCIONES CON PARA
}

1.1. En la interpretación que hace Manzini del infinitivo en las oraciones de propósito ("purpose") en inglés, se ofrecen dos diferentes categorías que están basadas en las relaciones de correferencia que se pueden establecer entre el infinitivo y el/los argumento(s) de la oración matriz ${ }^{1}$. Las estructuras son:

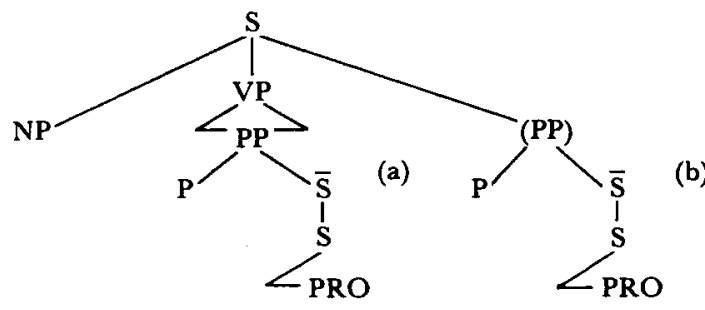

La correferencia del infinitivo es distinta en cada una de estas estructuras. En (a), donde la subordinada se enlaza directamente a la FV y está ligada a ella por un verbo que actúa como subcategorizador, el infinitivo puede ser correferente con el sujeto u objeto de la oración matriz. En (b), en la que el enlace no se hace directamente a la FV, sino que queda independiente de la FV, el inifinitivo es correferente únicamente con el sujeto de la oración matriz.

Esta interpretación formalista de Manzini nos brinda un acercamiento general a los problemas de correferencia del infinitivo

${ }^{1}$ M. R. MANZINI, “On control and control theory", $L I, 14$ (1983), 421446 , presenta una nueva teoría de control que unifica en una sola categoría a PRO y la anáfora léxica; para ello se vale de la "domain-governing category" que establece la línea divisoria entre las posiciones en que PRO será un elemento ligado y aquellas donde aparecerá como libre. De la exposición de Manzini tomamos, para nuestro caso, la interpretación y estructura que da 
y, con ello, nos provee una base para el análisis de la variación modal de estas estructuras en español. Nuestro trabajo intenta complementar esta interpretación desde otras perspectivas de análisis, en las cuales se tengan en cuenta los factores pragmáticos y semánticos que envuelven las distintas expresiones del discurso². Particularmente quiere describir el uso que el hablante hace del infinitivo en estas estructuras y calibrar las posiblidades de alternancia que presenta con el subjuntivo. Precisamente esta alternancia ha sido el caballo de batalla en los análisis empíricos sobre este tema. Partimos de la base de que la diferencia establecida entre estas estructuras repercutirá, indudablemente, en la posibilidad que se presenta al hablante para una selección del modo.

Tradicionalmente la gramática ha señalado que las oraciones finales alternan ambos modos dependiendo de la coincidencia o

de las oraciones a propósito ("purpose") en inglés. Nos dice: "Finally consider the case of a PRO subject of a modifier sentence. This case involves the configuration in (35), where the modified sentence attaches to VP or to S:

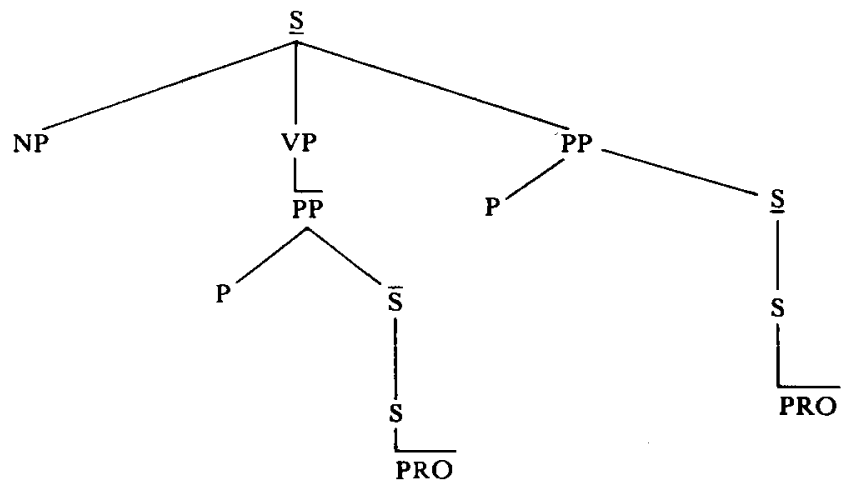

In (35) $\underline{\mathrm{S}}$ is the governing category of domain of PRO and $\underline{\mathrm{S}}$ contains a nominal accessible to $P R O$, hence $\underline{S}$ is the domain-governing category of PRO and PRO is bound in $\underline{S}$ by (22). In the case $\underline{S}$ is attached to VP the result is that PRO is coreferential with either an object or the subject of $\underline{S}$; which is the case with purpose sentences as in (36):

(36) John hired Mary [PRO to fire Bill].

In the case $\underline{S}$ is attached to $\underline{S}$, on the other hand, the result is that PRO is coreferential with the subject of $\underline{S}$; which is the case with purpose sentences as in (37) and eventually with purpose sentences as in (36):

(37) John fired Mary [PRO to hire Bill]'.

2 N. ChOмsкy, Lectures on government and binding, Foris, Dordrecht, 1981, p. 77, hace referencia a este posible aspecto del análisis cuando señala, mencionando a Manzini, que en ciertas oraciones de propósito "semantic or pragmatic considerations are operative". 
diferencia de sujetos que exista entre la subordinada y la oración matriz. Así lo repiten los autores más conocidos ${ }^{3}$; sirvan de ejemplo estas palabras de Gili Gaya que recogen una interpretación muy difundida:

las oraciones finales llevan el verbo en subjuntivo a causa del sentido de deseo o indeseo que encierra siempre el fin o intención con que se realiza un acto. Pero cuando el verbo principal y el subordinado tienen el mismo sujeto, este último va en infinitivo4.

1.2. Ya es un lugar común señalar que estas reglas de aparición del infinitivo basadas únicamente en la coincidencia de sujetos sólo sirven para caracterizar los casos más generales. Las descripciones y conteos llevados a cabo sobre el español son buena prueba de que existen casos que, con más abundancia de la que los textos gramaticales predican, no se sujetan a estas reglas ${ }^{5}$. Constituye un hecho repetido la constatación de construcciones que no permiten la alternancia de ambos modos en contextos formales donde serían aceptables. La alternancia infinitivo $v$ s. subjuntivo en las construcciones con para no es sino un ejemplo más del hecho ya tan repetido de que el funcionamiento de las lenguas es bastante más complejo del que demuestran algunas formalizaciones gramaticales. Si bien es verdad que los rasgos estructurales pueden ofrecer parámetros generales que dan razón de muchos de los hechos del español, con todo, se quedan cortos para medir toda la casuística que presenta el discurso. Los enlaces referenciales que se establecen en cada secuencia, como vimos en la interpretación de Manzini, tienen un papel relevante en su caracterización estructural pero el hecho es que, a su vez, estos enlaces

${ }^{3}$ Cf. J. Roca Pons, Estudios sobre perifrasis verbales del español, CSIC, Madrid, 1958; RAE, Esbozo de una nueva gramática de la lengua española, Espasa-Calpe, Madrid, 1974 y J. Alcina y J. M. Blecua, Gramática española, Ariel, Barcelona, 1975.

${ }^{4}$ S. Gili Gaya, Curso superior de sintaxis española, Bibliograf, Barcelona, 1964, p. 295.

${ }^{5}$ Véanse, entre otros, M. ARjona, "Peculiaridades sintácticas de las oraciones infinitivas finales", VII Congreso Internacional de ALFAL, Santo Domingo, República Dominicana; P. BENTIVOGLIO, "Clauses introduced by the preposition para in spoken Spanish: an analysis from a discourse viewpoint", Studies in Romance languages, Foris, Dordrecht, en prensa; y J. Moreno de Alba, "Algunas concurrencias sobre el infinitivo y el subjuntivo en la hipotaxis del español hablado en México", Actas del III Congreso de ALFAL, Instituto de Lingüística, San Juan, Puerto Rico, 1976, pp. 207-214. 
responden, muchas veces, no sólo a la estructura oracional que conforman sino a la secuencia referencial que se ha establecido en todo el párrafo.

Pasaremos primero a explicar lo que queremos decir en este último caso. En los análisis de texto nos encontramos con $\mathrm{mu}$ chos ejemplos de oraciones cuyos enlaces referenciales parecen provenir de elementos externos a la propia oración. Sirvan de muestra las oraciones:

(1) y me decía cómo adelantar para sacarme la placa del pecho

(2) le dije que me diera aquella casita a mi papá para irme allí

(3) yo hice que ella fuera al dispensario para que ella viera las condiciones ${ }^{6}$.

En estos ejemplos los controles referenciales que obran en las estructuras oracionales parecen provenir de argumentos que se encuentran fuera de ellas. Es un hecho lingüístico que se ha documentado en análisis anteriores y, en algunos de estos casos, la gramática formal ha hablado de unas transformaciones especiales, Super- $X$, que operan en determinadas construcciones poseedoras de unos verbos especiales que ejercen ese control ${ }^{7}$. En nuestro caso los verbos decir y hacer son los que dictan la referencia con "para irme allí" y "para que ella viera las condiciones". A título de ejemplo, una oración como:

(4) mi papá me dio aquella casita para que me fuera allí es una oración que puede alternar con:

(4') mi papá me dio aquella casita para irme allí

pero en la (2) decir, el verbo superordinado más alto, impone la coincidencia de sujetos y hace anómala la construcción en subjuntivo:

${ }^{6}$ P. Bentivoglio, art. cit., p. 13 (esta oración es del corpus venezolano).

${ }^{7}$ Esta transformación la utiliza J. T. Grinder ("Super equi-NP deletion", en Papers from the sixth Regional Meeting, Chicago Linguistic Society, Chicago, 1970, p. 313) para explicar los fenómenos gramaticales que han quedado fuera de las reglas de Rosembaum. Específicamente se refiere a casos como el de la oración siguiente, en que el infinitivo tiene el "contralor" en la oración más alta: "Harry thought that it would be difficult to leave". Son casos de antecedentes de larga distancia (K. P. Mohanan, "Remarks on control and control theory", $L I, 16,1985,637-648)$. 
? (2') le dije que me diera aquella casita a mi papá para que me fuera allí.

La oración (1) es ambigua en cuanto a si el sujeto de "para sacarme la placa del pecho" es el sujeto o el objeto de decir. Con el verbo en subjuntivo la lectura más obligada es la segunda, debido al control de decir, cuyo sujeto es él:

(1') y (él) me decía cómo adelantar para que (yo) me sacara la placa del pecho.

Esta correferencia con argumentos que han aparecido antes en el discurso se observa también en secuencias neutras, menos trabadas en cuanto a enlaces referenciales, donde lo que está en juego es una línea continua de secuencia referencial del párrafo (secuencia de tópico) ${ }^{8}$ :

(5) Nosotros acostumbrábamos a ir todos juntos al río, nos subíamos a los árboles, nos bañábamos... Recuerdo esos años de infancia con mucho cariño. Todo era como un tesoro, para mirar los troncos habia que hacer fila...

(6) Nosotros estábamos a razón de crear un curso aquí que integre lingüística, antropología y física. O sea que no sea un... tres o cuatro semanas de cada cosa sino tomar un problema general: evolución y adaptación de la especie humana. Para lograr una visión integradora es necesario mucho esfuerzo.

Como se observa en estas oraciones, el párrafo tiene un tópico establecido que es de lo que se está hablando, nosotros. Sin embargo, en todas ellas la subordinada donde aparece el infinitivo está ligada a una oración matriz que no repite los sujetos -en los dos últimos ejemplos son impersonales. Cabría, por lo tanto, la interpretación de sujeto arbitrario del infinitivo y seguramente ésta es la interpretación que una gramática formalista favorecería. Pero cabe también la interpretación de que los infinitivos en

${ }^{8}$ Según T. Grvon, On understanding grammar, Academic Press, New York, 1979, el tópico es una noción funcional del discurso, a diferencia del sujeto, que es sintáctico-gramatical. Distingue entre tópico de todo un párrafo, que define como el tema-tópico-sujeto de la conversación o discurso en el nivel del párrafo, y el tópico oracional o "sentential topic", que es el tema o sujeto de las predicaciones verbales expresadas dentro de cada cláusula. En "La vi la semana pasada" el sujeto-tópico yo está elidido pero la puede ser el tópico del párrafo. 
estas oraciones tienen como referente el tópico del párrafo, como se ve en la oración (5) y, posiblemente, en la (6).

2.1. Pasemos ahora a los análisis de los enlaces referenciales de la propia estructura y, con ello, a la variación modal que ofrecen. Por de pronto, en muchas construcciones se hace imposible la caracterización inmediata de estructura (a) o (b) debido a la ambigüedad que presentan. Sirvan de ejemplo las oraciones (7), (8), (9) y (10):

(7) Vino para llevársela.

(8) Contrató a Luis para reparar el tejado.

(9) Habló con el médico para ayudar a su hermano.

(10) Hemos escrito la carta para aclarar el malentendido.

2.1.1. La oración (7) no permite alternancia de modo si se mantienen los mismos enlaces referenciales. Se usa el subjuntivo cuando hay cambio de referente:

(7') Luis vino para que Luis se la llevara.

Según la clasificación que Solé ${ }^{9}$ hace de las estructuras con para del español, esta oración pertenece a la categoría "infinitive as modifier" con función adjetiva-adverbial. En inglés, según la autora, sería equivalente a una construcción de infinitivo con "(in order) to"':

(7) Vino para llevársela = "He came to take her with him".

Junto a ésta aparecen en la misma categoría de "modifier":

(11) Es buen sitio para descansar.

(12) Estoy listo para ayudarte.

Esta interpretación de Solé, en cuanto a entender el infinitivo como modificador indica, ya de base, que entre el infinitivo y la entrada léxica que le antecede (nombre, adjetivo o verbo) se establece un enlace estructural que está más cerca del tipo (a) que del (b). Precisamente por estar estas estructuras tan directamente li-

${ }^{9}$ Cf. Y. R. Solé y C. A. Solé, Modern Spanish syntax, Heath, Lexiton, MA, 1977, p. 91. 
gadas a la entrada léxica que les antecede se hace muy difícil el traslado a una posición inicial en la oración:

(11) ? Para descansar es buen sitio.

(12) ? Para ayudarte estoy listo.

Igualmente rechazan, o por lo menos no aceptan plenamente, en todos los casos, la construcción flexiva con sujeto diferente:

(11') ?Era buen sitio para que descansáramos.

$\left(12^{\prime}\right)$ *Estaba lista para que Luis ayudara.

Sí que lo permite, en cambio:

(7') Vino para que nos la lleváramos.

No parecen aceptar tampoco la falta de concordancia temporal:

$\left(11^{\prime \prime}\right)$ *Era/fue buen sitio para que descansemos.

(7') ?Vino/venía para que nos la llevemos.

2.1.2. La oración (8) es un caso típico de estructura (a) cuando se selecciona la opción del subjuntivo. Cuando está en infinitivo permite tanto una interpretación de estructura (a) como de (b). En esta última los sujetos son coincidentes con un significado similar a:

(8') Contrató a Luis para (poder) revisar el tejado.

que no tiene ya la interpretación de que Luis revisa el tejado, como en:

(8') Contrató a Luis para que revisara el tejado

De hecho, la oración (8') podría darse en un contexto como el siguiente:

(8') Contrató a Luis para poder revisar el tejado. Luis cuidará de los niños mientras él sube al tejado.

Asimismo esta interpretación de (8') permite la anteposición de la construcción con para: 
(8') Para poder revisar el tejado contrató a Luis.

La lectura (a) en esta oración establece vínculos más estrechos entre la oración matriz y la construcción con para. Se demuestra en la menor disposición a la anteposición:

(8") ?Para que revisara el tejado contrató a Luis.

(8) ?Para revisar el tejado contrató a Luis [en lectura (a)].

2.1.3. La oración (9) ofrece la particularidad, que no tiene la (8), de presentar una lectura única cuando aparece en infinitivo, la (b):

(9) Habló con el médico para ayudar a su hermano

que permite la anteposición:

(9) Para ayudar a su hermano habló con el médico

que podría ir seguida del contexto:

(9) Para ayudar a su hermano habló con el médico y le explicó la situación.

El cambio a subjuntivo conlleva el paso a una estructura (a):

(9') Habló con el médico para que ayudara a su hermano.

Aquí la interpretación es algo similar a "Le pidió al médico que ayudara a su hermano". La oración (9') tiene todos los rasgos de la oración (a) con dos argumentos en la oración matriz, y el objeto, aquí una FP, pasa a ser el sujeto del subordinado.

\subsubsection{En la oración:}

(10) Hemos escrito la carta para aclarar el malentendido

se permite la anteposición (que conlleva el verbo poder sobreentendido):

(10') Para (poder) aclarar el malentendido hemos escrito la carta.

Con todo, una interpretación (a) no es imposible, sobre todo cuan- 
do aparece en forma flexiva (aunque no exista un argumento en la oración matriz que pueda servir de sujeto de la subordinada):

(10") Hemos escrito la carta para que Luis aclare el malentendido.

En (10') el argumento podría estar sobreentendido:

(10') Hemos escrito la carta (a Luis) para que aclare el malentendido.

La diferencia entre (8) y (10) está en los rasgos de subcategorización de los verbos; contratar exige objeto + [humano] y finalidad con para; escribir, como cualquier verbo transitivo, puede llevar objeto directo e indirecto y la construcción con para es ya una construcción adicional de propósito.

En todos los ejemplos (salvo los del apartado 2.1.1) la forma de infinitivo puede interpretarse como posibilidad o potencialidad, que responde a una estructura menos trabada que la propia de cada caso.

2.1.5. Hemos estado observando cómo oraciones similares en términos de secuencias de entradas léxicas pueden ofrecer interpretaciones diferentes en términos de su estructura. Hay un grado mayor de dependencia en todos los casos en que la forma verbal pasa de infinitivo a la forma flexiva en subjuntivo. Todo parece indicar, pues, que la referencia, efectivamente, está íntimamente ligada a la estructura formal y, en cierto sentido, la determina cuando ésta se encuentra dentro de ciertos límites.

A la luz de la ejemplificación que hemos manejado, la estructura de las construcciones con para podría representarse en espanol con tres diagramas. La diferencia básica estribaría en la posibilidad de que la FP que representa la estructura con para estuviera: (I) bajo una FN (predicado nominal o sujeto), [oración (7)]; (II) bajo una FV [oración (8) o (10)] y (III) bajo O [oración (8'), (9) y $\left.\left(10^{\prime}\right)\right]$. Estas estructuras podrían representarse en los tres diagramas siguientes:

(I)<smiles>[18O]C[18O]</smiles><smiles>[AlH2]CP</smiles> 
(II)

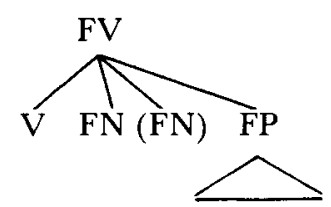

contrató a Luis para revisar el tejado; hemos escrito la carta para aclarar...
(III)

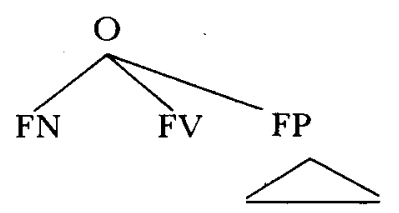

para (poder) ayudar a su hermano habló con el médico

En términos de su función semántica, la estructura (I) es la complementación de un nombre o adjetivo, la (II) es una construcción de finalidad, la (Ilb) de propósito y la (III) tiene implícita la idea de posibilidad, deseo o condición de que algo se lleve a cabo.

No vemos aún muy claro si la construcción de régimen directo de un verbo [oración (12)] pertenece a la (I) o la (II); la mayor libertad en términos de concordancia temporal parece indicar que está más cerca de la (II) que de la (I), con lo cual diferiríamos de Solé en términos de que pueda tratarse también en este caso de un infinitivo "modifier".

Establecer los límites entre una estructura y otra es una empresa difícil pues, como en otros aspectos de la sintaxis, los rasgos semánticos de las entradas léxicas tienden a determinar su propia estructura. Nos encontramos ante un espectro de variación gradual en el cual, por lo menos en el primer acercamiento, cualquiera de los límites que se quieran establecer en los miembros correspondientes a cada una de estas estructuras tiene que ser provisional.

2.2. Para comprobar todo esto pasemos a otros rasgos relacionados con estas estructuras, como los propios rasgos semánticos del sujeto del infinitivo. La mayor o menor especificación del referente puede también alterar la selección del modo. Ya es un hecho comprobado que a mayor arbitrariedad del referente, mayor posibilidad de aparición del infinitivo ${ }^{10}$. Si quisiéramos formalizar estas diferencias posibles en la impersonalidad de los referentes del discurso, el rasgo - [especificación] que correspondería a esa impersonalidad, podría, a su vez, estar matizado por el de $+/$ - [arbitrariedad], lo cual le permitiría formular el mayor o menor grado de arbitrariedad que puede llevar esa no especifica-

${ }^{10}$ P. Bentivoglio, art. cit., p. 11. 
ción. Según los ejemplos de nuestro texto, los diferentes matices en la arbitrariedad del sujeto de la subordinada ocasionan, también, restricciones en la alternancia modal.

Como comprobación de estas aseveraciones, observemos el comportamiento de ciertas formas impersonales: uno, tú, alguien, etc., que tienen referentes indeterminados y aparecen, muchas veces, explícitos como sujetos del infinitivo ${ }^{11}$.

En el ejemplo de la oración (13) el tú sujeto del infinitivo va acompañado de los rasgos - [especificado], + [arbitrario] puesto que el referente está totalmente indeterminado y puede ser el hablante, oyente o cualquiera:

(13) bueno ahora para tú abrir una cuenta en el Popular, hay una hoja...

En la oración (14), por el contrario, el uno, a pesar de ser un elemento inespecificado, se presenta como miembro de una clase determinada y, con ello, en oposición a otro grupo con el que se pone en contraste:

(14) siempre tenían una orquesta pequeña para uno bailar.

Los rasgos que les corresponden en este caso son - [especificado], - [arbitrario], puesto que se hace referencia al conjunto de empleados a quienes los jefes les ponían música en la fábrica durante las fiestas (en estos términos se distingue a los empleados de los jefes).

Ejemplos de - [especificado], + [arbitrario] son los unos de las oraciones (15) y (16), que presentamos a continuación:

${ }^{11}$ Se sabe que estas apariciones son esporádicas y constituyen usos que, en cierto sentido, están fuera de norma. Según el tercer modelo de la gramática generativa, en el infinitivo la posición de sujeto sólo puede ser ocupada por una categoría especial, PRO, que es pronombre y anáfora a la vez y que carece de matriz fonológica; cf. CHOMSKy, op. cit., y L. Rizzi, Issues in Italian syntax, Foris, Dordrecht, 1982, pp. 77 ss. Con todo, esta clase de sujetos y algunos otros pronominales con infinitivo adquieren cierta frecuencia en español, sobre todo en el español del Caribe (cf. entre otros, M. SUÑER, "Subjects of infinitives in standard and Caribbean Spanish", Cornell University, Ithaca, 1983, y A. Morales, "Infinitivo con sujeto expreso en el español de Puerto Rico", Gramáticas en contacto, Playor, Madrid, 1986). En inglés la explicación de tal aparición proviene de las características especiales de la preposición for que "gobierna" al sujeto del infinitivo. 
(15) para uno poder estudiar es necesario tener chavos

(16) y se tapaban bien tapaítos para uno tomar agua.

En todos los casos el hablante está incluido en uno, pero en la oración (14) el referente es "yo y el grupo al que pertenezco" mientras que en las oraciones (13), (15) y (16) es algo así como "yo y cualquiera"'12.

En la oración (14) el sujeto del infinitivo conlleva un cambio de referente, es una estructura con sujeto diferente al de la oración matriz; por eso puede alternar con subjuntivo:

(14') siempre tenían una orquesta pequeña para que uno bailara, que es una estructura similar a la de la oración (10). El cambio a infinitivo sin sujeto expreso altera totalmente la información (en este caso serían los jefes los que tendrían la orquesta para bailar ellos mismos):

(14') siempre tenían una orquesta para bailar.

Cabe señalar las distintas características que se observan en ambos tipos de construcciones cuando aparecen estas formas impersonales. Contrastando las oraciones (15) y (16), ejemplos de estructura (III), se observa que el paso a subjuntivo conlleva en la (16) una especificación referencial que no aparece en la (15).

(15') para que uno pueda estudiar es necesario tener chavos

(16') y se tapaba bien tapaíto para que uno tomara agua.

En (16') uno parece ir acompañado de un rasgo significativo adicional que le permite contrastar con el sujeto impersonal del verbo matriz, rasgo que parece colocarlo dentro de los lazos estructurales de una oración tipo (II), situación que no se da en la (15').

Así pues, la ambigüedad en cuanto a responder a estructura (II) o (III), que presentan muchos de los casos encontrados en los textos, se hace patente en los usos de las formas impersonales. La correferencia de la forma impersonal de la subordinada con

${ }^{12}$ Señala B. Lavandera en "Tensión entre lo impersonal y lo personal en la organización del discurso" (Variación y significado, Hachette, Buenos Aires, 1984, pp. 101-124) refiriéndose al español de Argentina: "A pesar de que en el nivel de la oración la diferencia semántica entre uno y vos-usted es bastante sutil, e incluso a veces imposible de percibir, cada una de estas formas se pueden utilizar, y de hecho se utilizan, al servicio de funciones discursivas que le son únicas"' (p. 123). 
la impersonal de la matriz sólo se mantiene en una estructura (III). En la estructura (II) la forma impersonal acompañada del infinitivo aporta unos rasgos de diferencia de sujetos que se hacen patentes en la forma flexiva de subjuntivo [oraciones (16') y (14')]. Cuando no hay referente en la oración principal la impersonalidad de la subordinada conlleva siempre una interpretación (III) en este tipo de construcciones:

(11) bueno ahora para tú abrir una cuenta en el Popular, hay una hoja...

2.3. El último rasgo en el que queremos insistir es el de la secuencia temporal de la construcción y, con ello, los distintos matices temporales que el infinitivo puede expresar. Según Alarcos, "el infinitivo, pues, es la forma verbal más general al expresar el proceso en reposo, sólo como simple potencia realizable". "Tiempo in posse" lo llama el autor más adelante, donde "la tensión (carga potencial de tiempo) está intacta, en el infinitivo no hay distensión"'13.

Pero el infinitivo ofrece matices en esta generalidad o virtualidad de acción; haciendo referencia al infinitivo de las completivas se postula que el tiempo del infinitivo debe ser recuperable por el hablante desde el verbo regente y se insiste en que cuanto menor es la libertad de selección de tiempo que el verbo de la matriz admite, mayor es la posibilidad de infinitivo ${ }^{14}$. Esto nos indica, según creemos, que puede existir alguna manifestación temporal aunque sea mínima. Podemos suponer que el infinitivo en español es capaz de ofrecer matices temporales mínimos, que se mueven en un espectro que va desde la no temporalidad (tiempo in posse) en nominalizaciones y lexicalizaciones hasta lo que podríamos llamar transparencia del tiempo del verbo principal en las completivas. En este último caso es cuando las marcas temporales débiles le permiten al infinitivo alternar con el subjuntivo ${ }^{15}$.

13 Cf. E. Alarcos Llorach, Gramática estructural, Gredos, Madrid, 1969, pp. 104-105. Estas mismas características aparecen recogidas en posturas teóricas más actualizadas, que para dar cuenta del infinitivo recurren a los rasgos -Tense -AGR en la formalización del Auxiliar.

${ }^{14}$ Los verbos que ofrecen menor posibilidad de selección son los de la categoría de suasión y similares: atreverse, prometer, prohibir, vacilar, conseguir, abstenerse, etc. En éstos se da, por ello mismo, la verdadera alternancia con subjuntivo cuando los rasgos referenciales lo permiten.

${ }^{15}$ Esta variabilidad se refleja en la postura de algunos estudiosos como, 
En nuestra investigación, que se limita a las construcciones con para, el abanico de variación temporal del infinitivo es muy limitado. La mínima expresión temporal se da en las estructuras con referente arbitrario en el infinitivo:

(17) se necesita mucho coraje para hablar con el jefe

(18) para sobresalir en la vida hay que fajarse.

Estas construcciones permiten, con más facilidad que otras, la intercalación del verbo poder; es donde el infinitivo muestra más su atemporalidad o tiempo in posse. En ellas los infinitivos llevan la idea de posibilidad o futuridad implícita, donde lo pertinente son los propios rasgos léxicos del verbo utilizado y no su posible acción en el decurso. En estas construcciones es donde, a nuestro entender, la alternancia modal no es posible si se mantiene el significado de potencialidad o futuridad de acción, o sea de verbo entendido como conjunto de rasgos semánticos. La forma flexiva conlleva una especificación referencial que no encaja con la abstracción implícita de las estructuras. En este caso, de haber cambio modal es cuando, según lo entendemos, la variación en el modo vendría como consecuencia de una selección voluntaria del hablante a una mayor especificación referencial y temporal que, incluso, en algunas de ellas ocasionaría un cambio de estructura como en (16).

La manifestación temporal más patente en esa gradación de ausencia temporal, que es la que corresponde al "reflejo" o "transparencia" temporal, se da en la estructura (II), en que la dependencia oracional es más trabada y en la que, por lo tanto, las estructuras de para guardan más semejanza con las completivas que alternan estos modos ${ }^{16}$. Se caracterizan porque el referente apa-

por ejemplo, C. PICALlo, "The INFL-node and the nuil subject parameter", $L I, 15$ (1984), 75-102, que ha llegado a establecer que el subjuntivo, como el infinitivo, carece de rasgos temporales. Según la autora (pp. 82 ss.) las oraciones de subjuntivo comparten propiedades de infinitivo e indicativo. Como infinitivo puede aparecer sólo en posiciones subcategorizadoras y nunca en "root sentences". Como indicativo el sujeto de un subjuntivo puede ser léxico o no realizado fonológicamente. Para la autora el subjuntivo no tiene marca de $+1-$ pasado; esta marca depende de la oración principal, por lo que este modo está, en cuanto a variación tamporal, más restringido que el indicativo.

16 Nos referimos, otra vez, a los verbos de suasión que, por imponer restricciones temporales fuertes al verbo subordinado, permiten con mayor libertad que otros verbos la alternancia modal, cf. K. SAUER, Sentential complementation in Spanish, University Microfilms, Ann Arbor, MI, 1977. 
rece generalmente marcado con el rasgo + [especificado]. En ellas es posible la libre alternancia, el cambio de modo no conlleva cambio de estructura.

Como conclusión, insistimos en que todo lo expuesto hasta ahora parece indicar que estas construcciones con para se deben caracterizar atendiendo a tres factores fundamentales: las estructuras que forman; los enlaces referenciales que se establecen entre los argumentos de la subordinada y matriz; y la mayor o menor marca temporal que manifiesta el verbo subordinado. Los tres factores son interdependientes y permiten gradación en sus propiedades. Estas gradaciones se podrían representar en tres niveles diferentes:

\begin{tabular}{llll}
\hline & Estructura & \multicolumn{1}{c}{ Referencia } & \multicolumn{1}{c}{ Tiempo } \\
\hline 1 & (I) & suj. específico & marca débil (subj.) \\
2 & (IIa y b) & suj. espec. - arb. & trans. tiempo (inf.) \\
3 & (III) & suj. espec. + arb. & tiempo in posse (inf.) \\
\hline
\end{tabular}

La unión de estos rasgos no es totalmente libre o, mejor dicho, lo es dentro de ciertos límites. Las estructuras (I) y (II) se combinan con marcas temporales débiles - lo que hemos llamado "transparencia" de tiempo-y, generalmente, con sujetos no arbitrarios.

La estructura (III) se asocia con las marcas temporales más débiles y la mayor arbitrariedad en los sujetos aunque su rasgo más pertinente parece ser el de evitar el contraste de referentes. Por ello permite también los sujetos específicos, siempre que sean coincidentes ("Para poder reparar el tejado contrató a Luis").

El infinitivo es polisémico en (IIa). En una de sus lecturas está en variación libre con el subjuntivo ("Contrató a Luis para reparar [que reparara] el tejado"), en la otra constituye una lectura diferente porque representa una estructura distinta, la (III). En ésta no tiene alternancia: "Contrató a Luis para (poder) reparar el tejado", similar a "Para (poder) reparar el tejado contrató a Luis".

Para que la estructura (III) pueda pasar a subjuntivo se necesitan rasgos muy particulares. Sería el caso de la oración (15), que tiene un contexto que impide la ambigüedad:

(15') Para que uno pueda estudiar es necesario tener chavos, que con otros referentes se podría construir con sujetos específicos, incluso: 
(15') Para que Luis pueda estudiar su madre necesita tener chavos, (que, en sí, es ya otra oración). En esta estructura es posible, en cambio, la libre alternancia con subjuntivo con sujetos iguales:

(15"') Para que Luis pueda estudiar necesita tener chavos.

El cambio de modo en esta estructura está menos regulado que en las otras. La estructura (IIb) tiene también infinitivo polisémico: "Hemos escrito la carta (a Luis) para aclarar el malentendido". En la lectura de estructura (IIb) alterna con subjuntivo siguiendo el patrón establecido por Gili Gaya y la gramática tradicional; la diferencia de sujetos hace aparecer el subjuntivo: " $\mathrm{He}$ mos escrito la carta para que aclaren el malentendido"' o " $\mathrm{He}$ mos escrito la carta a Luis para que aclare el malentendido".

Así pues, nos encontramos ante tres tipos de alternancia subjuntivo-infinitivo que, en sí, son complementarias:

El primero, que llamaremos de variación libre, es el que se da en determinadas construcciones de para que establecen con el verbo de la oración matriz un enlace especial similar al de los verbos de suasión. Son nuestros casos de estructura IIa.

El segundo, que llamaremos de alternancia tradicional, son los casos en que el cambio de modo obedece a la semejanza o diferencia de sujetos. Estructura IIb y el ejemplo de la oración (7).

El tercer tipo es diferente, aquí intervienen factores de otra clase. El hablante selecciona el infinitivo cuando no quiere marcar un evento sino la potencialidad o, más bien, los rasgos semánticos de la entrada léxica representada por el infinitivo. Son los casos de la estructura III. Nosotros la llamamos pragmática, porque en la selección de modo intervienen factores no necesariamente sintácticos.

Amparo Morales 